\title{
The Zurich magnetic resonance imaging protocol for standardized staging and restaging of sinonasal tumours*
}

\author{
Christian M. Meerwein', Athina Pangalu², Shila Pazahr, Lorenz Epprecht', \\ Michael B. Soyka', David Holzmann' \\ ' Department of Otorhinolaryngology, Head and Neck Surgery, University Hospital Zurich, University of Zurich, Switzerland \\ 2 Department of Neuroradiology, University Hospital Zurich, Zurich, University of Zurich, Switzerland \\ ${ }^{3}$ Department of Radiology, Balgrist University Hospital, Zurich, University of Zurich, Switzerland
}

Rhinology Online, Vol 4: 161 - 164, 2021

http://doi.org/10.4193/RHINOL/21.038

*Received for publication:

August 8, 2021

Accepted: August 20, 2021

Published: August 28, 2021

\section{To the Editor:}

In combination with paranasal sinus computed tomography (CT), cross-sectional imaging with magnetic resonance imaging (MRI) is mandatory for staging and restaging of primary sinonasal malignancies ${ }^{(1,2)}$. In the initial staging, MRI defines tumour size, provides information on extension into adjacent compartments of the sinonasal tract (in particular orbit, anterior or middle cranial fossa, leptomeningeal and brain parenchyma) and consecutively helps to determine the clinical T category. Furthermore, MRI delineates tumour from surrounding tissue (e. g. retention of mucus, reactive polyps) and may even identify perineural spread and bone marrow infiltration ${ }^{(3,4)}$. The signal intensity of tumours varies depending on their cellularity, mucin content and presence of hemorrhage. However, even state-of- the-art cross-sectional imaging may fail to correctly identify orbital or skull base infiltration. Thus, both, false-positive and false-negative findings must be considered. Common pitfalls particularly include 1) the discrimination of bony pressure erosion and bony infiltration of the anterior skull base or the medial orbital wall and 2) the discrimination of reactive dural enhancement and dural infiltration by tumour ${ }^{(5,6)}$. Based on these difficulties and in analogy to upper aero-digestive tract squamous cell carcinomas, we recently suggested an obligatory exploration of all sinonasal tumours under general anesthesia and targeted biopsy, if necessary ${ }^{(7)}$.

Besides its role in the initial staging (Figure 1), MRI is also important in the restaging setting, where tumour persistence or recurrence and treatment-associated alterations may be challenging

Table 1. Standardized MRI protocol for staging and restaging of sinonasal tumours.

\section{MRI sequence}

- T1-weighthed, native, coronar section

- T1-weighted, native, transverse section

- T2-weighted, native, fat-suppressed, coronar section

- T2-weighted, native, transverse section

- Diffusion-weighted, native axial section

- T1-weighted, contrast-enhanced, fat-suppressed (FS), transverse section

- T1-weighted, contrast-enhanced, fat-suppressed (FS), coronar section

- T1-weighted, contrast-enhanced, fat-suppressed (FS), sagittal section

\section{Characteristics}

TR / TE 650/9.5ms, FOV 180, NEX 1, slice thickness 3.0mm, distance between slices $0.3 \mathrm{~mm}$ - Fat: bright, due to its short relaxation time, high contrast towards other tissues

- Tumour: longer relaxation time, poor contrast of tumour against muscle - Mucosa: similar to tumour

Fast Spin Echo-Sequence, 5100/89 ms, FOV 180, NEX 2, slice thickness 3.0mm, distance between slices $0.3 \mathrm{~mm}$. Different techniques for fat suppression (e.g. IR or for less metallic artefacts DIXON).

- Fluid: bright

- Tumour: high contrast of tumour towards muscle, poor contrast of tumour towards fat - Fat: dark in fat-suppressed sequences, which allows a good contrast between tumour and suppressed fat

Diffusion weighted images are helpful in differentiating malignant tumours from benign based on ADC values

\section{Gadolinium chelate contrast agents}

- Sagittal plane best for assessment of potential bony or dural infiltration of the skull base

- Assessment of bone (anterior skull base) with dark signal adjacent to bright signal of nasal mucosa

MRI, magnetic resonance imaging. FOV, field-of-view; NEX, number of excitations; TE, echo time; TR, repetition time. 

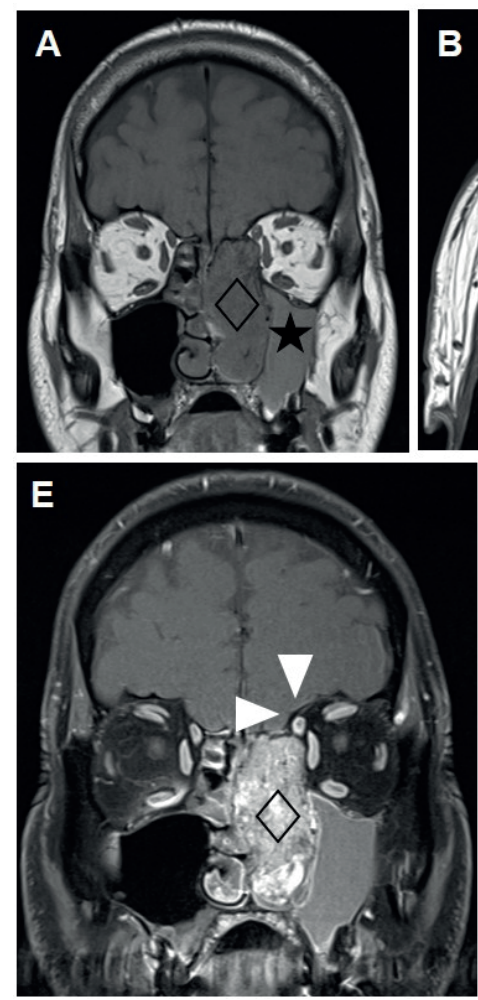
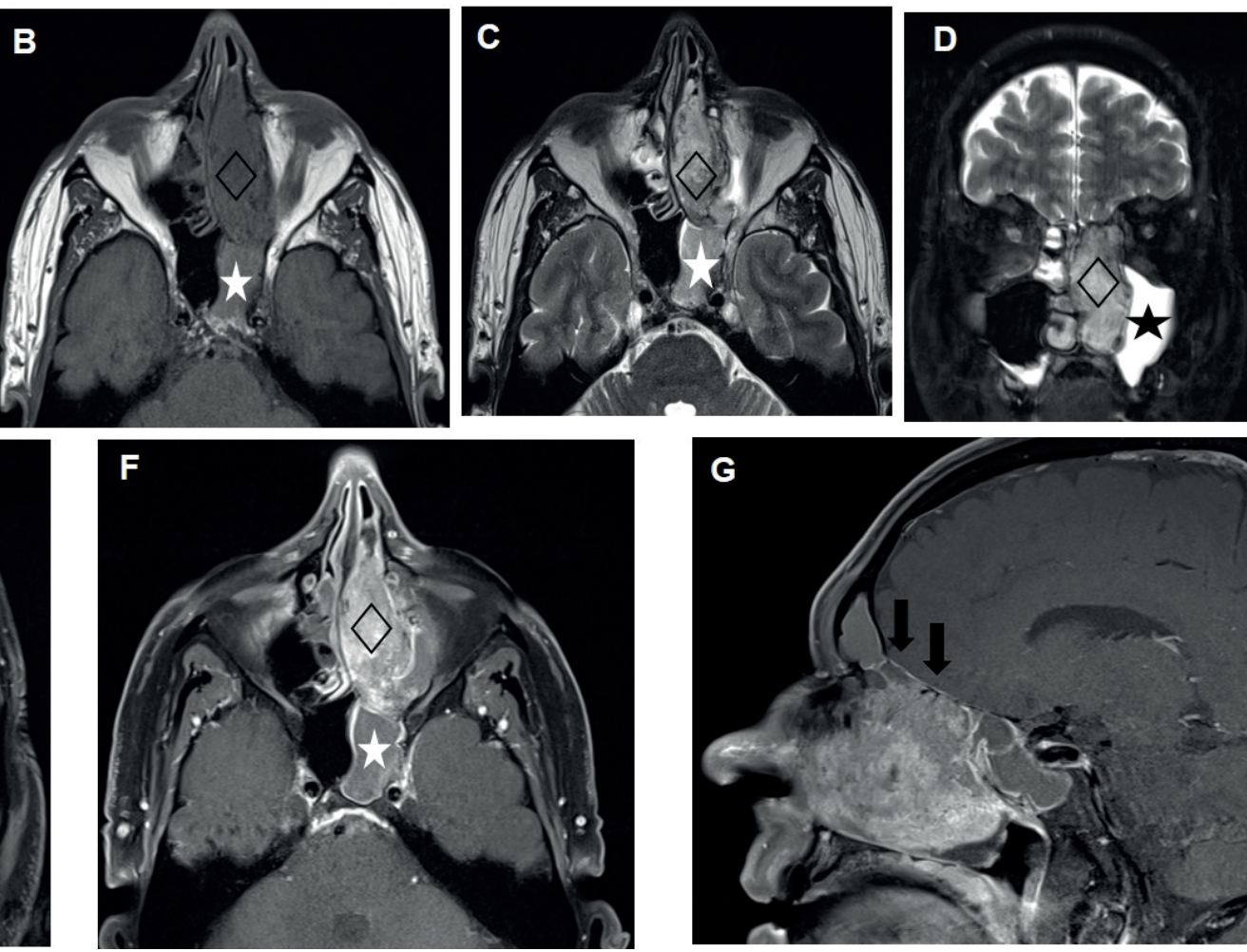

Figure 1. Magnetic resonance imaging for initial staging of a 55-year old male patient with a biopsy-proven sinonasal undifferentiated carcinoma (SNUC). On T1-weighted, non-enhanced coronar (A) and transverse (B) sections, we see a heterogeneous, mostly hypointense, obstructing and poorly demarcated tumour in the nasal cavity and in the adjacent ethmoidal cells (black rhomb), with secondary opacification of the MS (black asterix) and the SS (white asterix) due to mucus retention. On T2-weighted, non-enhanced transverse sections (C), the tumour (black rhomb) displays an inhomogeneous, intermediate signal (white asterix, SS). On T2-weighted, FS coronar images (D), the tumour (black rhomb) is easy to differentiate from mucus retention in the MS (black asterix). As best seen on T1-weighted, CE FS coronar (E) sections in combination with T1-weighted non-enhanced coronar sections (A), a clear distinction between erosion and infiltration of the bony orbit and periorbita in its supero-medial quadrant in proximity to superior oblique muscle is challenging (white arrowheads, (E)). However, no affection of the extraconcal adipose tissue or extraocular eye muscles was suspected. On T1-weighted CE FS axial (F) sections, we can confirm an inhomogeneous tumour (black rhomb) in the nasal cavity and ethmoidal cells, with secondary opacification of the SS (white asterix). On T1-weighted CE FS sagittal sections (G), we see a linear and thin dural enhancement (black arrows), which is most likely reactive. Intraoperative exploration of the tumour revealed an infiltration of the periorbita in its supero-medial quadrant, without affection of the extraconal fat tissue or the extraconcal eye muscles. However, no evidence of infiltration of the bony or dural skull base was found. CE, contrast-enhanced; FS, fat-suppressed; MS, maxillary sinus; SS, sphenoid sinus.

to distinguish (Figure 2). Thus, a standardized way to compare MRI images at different time points is of utmost importance. In an effort to increase this comparability and in analogy to previous published protocols for paranasal sinus $\mathrm{CT}$, we aimed to establish a standardized MRI protocol for sinonasal malignancies at our institution, which allows to reliably compare imaging sessions, even on different scanners and at different sites. Aiming to achieve a high inter-patient and intra-patient comparability, this protocol should remain unchanged, regardless (A) the tumour entity, (B) the tumour origin (nasal cavity / ethmoid sinus vs maxillary sinus), (C) the treatment algorithms or (D) the timing of the examination (pretherapeutic vs. posttherapeutic). Table 1 provides details on the proposed MR sequences.
Assessment of the bony and dural anterior skull base In combination with CT imaging, an adequate assessment of the bony and dural skull base is pivotal. Eisen et al. stated, that the presence of pial enhancement, focal dural nodules or dural thickening of more than $5 \mathrm{~mm}$ is highly accurate in predicting dural invasion, while linear dural enhancement may also be reactive (5). McIntyre et al., however, found that the presence of " $\geq 2 \mathrm{~mm}$ of dural thickening," "loss of hypointense zone on T1-weighted images", and "nodular dural contrast enhancement" were highly predictive for dural invasion ${ }^{(8)}$. As Schuknecht et al. stated, the bony skull base is best seen on T1-weighted, contrast enhanced, fat-suppressed series with a dark signal of the bone and an adjacent, bright signal of the nasal mucosa ${ }^{(1)}$. Fat suppression is needed to eliminate any high signal intensity from adjacent fat 

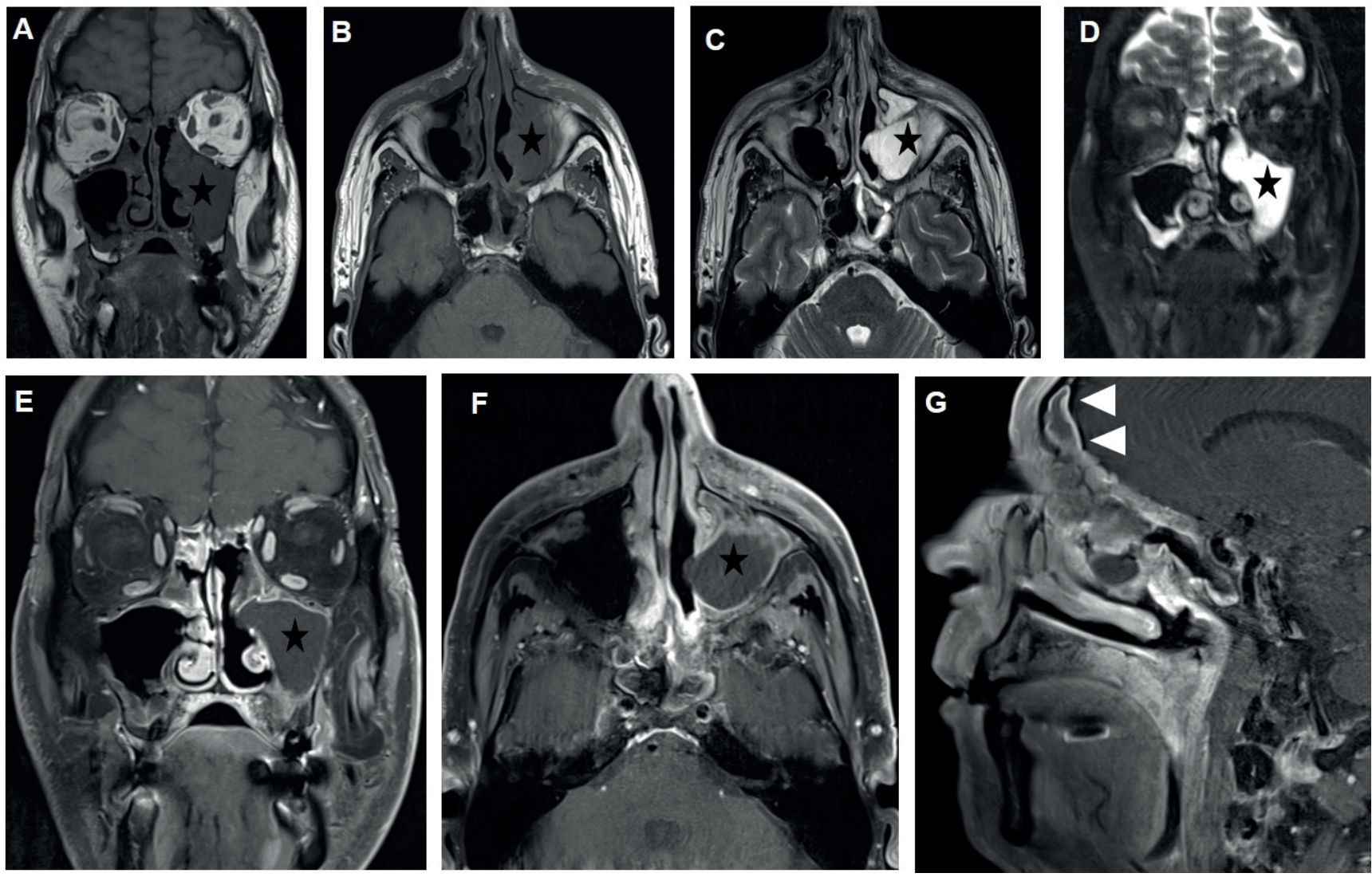

Figure 2. Magnetic resonance imaging restaging examination of the 55-year old patient from Figure 1, three months after a transnasal-transcribriform tumour resection and postoperative proton beam therapy for a sinonasal undifferentiated carcinoma (SNUC). On T1-weighted, non-enhanced coronar (A) and transverse (B) sections, we see a hypointense opacification of the maxillary sinus (black asterix) and partially of the nasal cavity. On T2-weighted, non-enhanced transverse (C) sections and T2-weighted, FS coronar (D) sections, the signal is hyperintense and corresponds most likely to mucus retention in the maxillary sinus (black asterix). On T1-weighted, CE FS coronar (E) and transverse (F) sections, we see a marginal contrastenhancement of the nasal mucosa, framing the postoperative cavity. The signal of the maxillary sinus (black asterix) is homogenous and hypointense. On T1-weighted, CE FS sagittal (G) sections, there is an inhomogeneous, T1 hypointense contrast enhancement with particular delineation around the frontal recess. The contrast enhancement is predominantly marginal (white arrowhead) and most likely reactive. No evidence of dural and or orbital affection. So far, the patient remained free of disease. CE, contrast-enhanced; FS, fat-suppressed; MS, maxillary sinus; $S$, sphenoid sinus.

(i.e., fatty marrow, etc.) that might be confused with or obscure actual enhancement.

\section{Assessment of the orbit}

Radiological criteria for determining orbital infiltration in MRI include (a) bony orbit and periorbita, (b) extraconal adipose tissue, (c) extraocular eye muscles and (d) intraconal structures ${ }^{(2,7)}$. However, as previously shown, one has to account for false区 positive findings and an overestimation of the true extent of infiltration ${ }^{(7)}$. The most important plane for extra- and intraconal structures is the coronal plane. As the lamina papyracea is thinnest directly posterior to the nasolacrimal duct, a careful attention in the coronal section must be paid in this distinct area. The coronal plane also allows detection of eye muscle infiltrations, extraconal extension, and infiltration of the optic nerve ${ }^{(9)}$. Healthy muscles have a low signal intensity in T1-weighted images, in comparison to the high signal intensity of the adipose tissue
(9). Intraconal masses can be visualized on unenhanced T1weighted sequences, because nearly all intraconal pathologies are hypointense in comparison to hyperintense surrounding adipose tissue ${ }^{(9)}$.

\section{Conclusion}

We here present a standardized and easy to reproduce MRI protocol for staging and restaging of sinonasal tumours, which allows a thorough assessment of the orbit and the anterior skull base. In order to achieve a high inter-patient and intrapatient comparability, this protocol should remain unchanged, regardless the timing of examination, tumour entity, treatment algorithms or tumour origin.

\section{Authorship contribution}

CMM: designed the study, collected data, designed the figures, 
wrote the manuscript; AP: neuroradiological expertise, wrote the manuscript, developed the concept; SP: neuroradiological expertise, wrote the manuscript, designed the figures; LE: edited the manuscript; MBS: edited the manuscript; $\mathrm{DH}$ : designed the study, edited the manuscript, developed the concept.

\section{Acknowledgments}

Not applicable.

\section{Funding}

None.

\section{Ethics approval and consent to participate}

Not applicable.

\section{Consent for publication}

Not applicable.

Availability of data and materials

Not applicable.

\section{Conflict of interest}

None.

\section{References}

1. Schuknecht B, Simmen D. State of the Art Diagnostic Imaging of Paranasal Sinus Disease. Laryngo-Rhino-Otologie. 2002;81(2).

2. Madani G, Beale T. J. LVJ. Imaging of sinonasal tumors. Semin Ultrasound, CT MRI. 2009;30(1):25-38.

3. Kimura $Y$, Sumi M, Sakihama N, Tanaka F, Takahashi H, Nakamura T. MR imaging criteria for the prediction of extranodal spread of metastatic cancer in the neck. Am J Neuroradiol. 2008;29(7)

4. Connor SEJ. The Skull Base in the Evaluation of Sinonasal Disease: Role of Computed Tomography and MR Imaging. Vol. 25, Neuroimaging Clinics of North America. 2015.

5. Eisen MD, Yousem DM, Montone KT, Kotapka MJ, Bigelow DC, Bilker WB, et al. Use of preoperative MR to predict dural, perineural, and venous sinus invasion of skull base tumors. Am J Neuroradiol. 1996;17(10).

6. Eisen MD, Yousem DM, Loevner LA, Thaler ER, Bilker WB, Goldberg AN. Preoperative imaging to predict orbital invasion by tumor. Head Neck. 2000 Aug;22(5).

7. Meerwein CM, Pazahr S, Soyka MB, Hüllner MW, Holzmann D. Diagnostic accuracy of computed tomography and magnetic resonance imaging compared to surgical exploration for anterior skull base and medial orbital wall infiltration in advanced sinonasal tumors. Head Neck. 2020;42(8)

8. Mcintyre JB, Perez C, Penta M, Tong L, Truelson J, Batra PS. Patterns of dura involvement in sinonasal tumors: Prospective correlation of magnetic resonance imaging and histopathologic findings. Int Forum Allergy Rhinol. 2012;2(4).

9. Lemke AJ, Kazi I, Felix R. Magnetic reso- nance imaging of orbital tumors. Eur Radiol. 2006 Oct 1;16(10)

Christian M. Meerwein, MD

Department of Otorhinolaryngology,

Head \& Neck Surgery

University Hospital Zurich

Frauenklinikstrasse 24

8091 Zurich,

Switzerland

Phone number: +41 442555850

Fax number: + 41442554556

E-Mail: christian.meerwein@usz.ch

ISSN: 2589-5613 / @2021 The Author(s). This work is licensed under a Creative Commons Attribution 4.0 International License. The images or other third party material in this article are included in the article's Creative Commons license, unless indicated otherwise in the credit line; if the material is not included under the Creative Commons license, users will need to obtain permission from the license holder to reproduce the material. To view a copy of this license, visit http://creativecommons.org/ licenses/by/4.0/ 